\title{
Generation of electrostatic oscillations in the ion cyclotron frequeny range by modulated ECRH
}

\author{
HP Laqua, D Moseev, P Helander, P Aleynikov, S Marsen, \\ NB Maruschenko, T Stange \\ Max-Planck-Institut für Plasmaphysik, Greifswald, Germany \\ E-mail: dmitry.moseev@ipp.mpg.de
}

\begin{abstract}
.
Ion cyclotron frequencies are generally three orders of magnitude (electronto-ion mass ratio) smaller than the electron cyclotron frequency range. Here we propose a novel way of generating electrostatic fluctuations along the magnetic field in the ion cyclotron frequency range by modulated electron heating in regions with a non-zero gradient of the magnetic field strength along the magnetic field line. A possible conversion mechanism of these electrostatic fluctuations into shear Alfvén waves is also presented.
\end{abstract}

9 July 2018

\section{Introduction}

Electron cyclotron resonance heating $(\mathrm{ECRH})$ is one of the most important methods of plasma heating in modern fusion research. It is distinguished from other means of wave heating by a particularly precise control over the power deposition. Moreover, depending on the plasma conditions and the chosen microwave frequency, different strategies can be adopted for achieving the most efficient way of heating or driving the current. A general property of microwave heating in the electron cyclotron frequency range is nearly a $100 \%$ coupling efficiency of the power into the plasma [1].

In this letter we propose a novel scheme of modulating the ECRH power by inducing beat-waves in the ion cyclotron frequency range, i.e. $10-100 \mathrm{MHz}$, see Section 2. In the past, power modulation by beat-waves used for ICRF heating have been used to excite Alfvén eigenmodes [2-4], but the physical mechanism of generating the resulting waves is different from that in our proposal. In Section 3 below we explain how modulated ECRH power can lead to the generation of electrostatic oscillations in the ion cyclotron frequency range. In Section 4 we discuss a possible conversion mechanism of these oscillations into waves, and in Section 5 we discuss diagnostic methods how to detect this effect. Section 6 concludes the paper.

\section{Fast power modulation of the microwave radiation}

Gyrotrons, the sources of microwave radiation used for ECRH, use kinetic energy of an accelerated electron beam in a magnetic field for emitting microwaves. The power of these microwaves is proportional to the acceleration voltage [5]. 


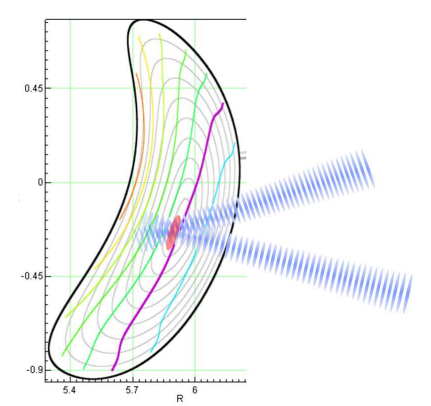

(a)

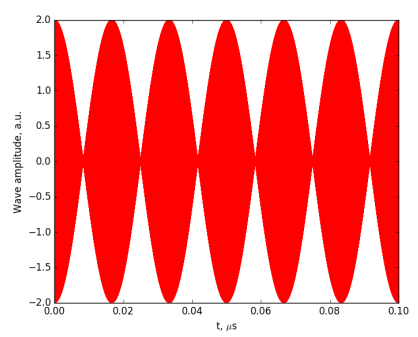

(b)

Figure 1. Illustration to the beat-wave generation by gyrotrons. (a) Microwave injection scheme for the beat-wave generation. (b) Beat wave created by two waves at frequencies $140 \mathrm{GHz}$ and $140.06 \mathrm{GHz}$, respectively. $60 \mathrm{MHz}$ of frequency difference translates into $30 \mathrm{MHz}$ beat wave.

Modulated ECRH requires an accelerating voltage modulator, which might be cumbersome to design for the $\mathrm{MHz}$ frequency range [6]. A modulated wave can however fairly easily be generated by letting two waves of fixed frequency beat against each other.

Gyrotrons are coherent sources of microwaves. One ECRH plant normally comprises several gyrotrons that work at similar frequencies. The exact frequency of a gyrotron is a function of its power and accelerating voltage, and can be varied by 10-150 MHz. Letting two gyrotron beams overlap at the absorption region will create a beat-wave pattern,

$$
\cos \omega_{1} t+\cos \omega_{2} t=2 \cos \frac{\omega_{1} t-\omega_{2} t}{2} \cos \frac{\omega_{1} t+\omega_{2} t}{2},
$$

where $\omega_{1}$ and $\omega_{2}$ are two gyrotron frequencies, respectively. The concept is also illustrated in Fig. 1. This obviously leads to the modulation of the ECRH power.

Although the amplitude of the envelope oscillates at half the frequency difference of two sources, power modulations will take place twice as often, at the frequency $\omega=\left|\omega_{1}-\omega_{2}\right|$. In the regular three-wave mixing, the resulting wave should also have $\omega=\left|\omega_{1}-\omega_{2}\right|$, however momentum conservation would also dictate a fixed wave vector: $\vec{k}=\overrightarrow{k_{1}}-\overrightarrow{k_{2}}$. This limitation does not apply to the beat-wave initiated process.

\section{Generation of electrostatic field by modulated heating}

The idea that the mirror force generates electric fields along the field line was initially suggested by Persson in 1963 [7]. Our proposed mechanism relies on the fact that ECRH in the extraordinary mode (X-mode) heats electrons in the direction transverse to the magnetic field. The increase of perpendicular energy of an electron leads (due to the conservation of magnetic momentum) to a change in the mirror force in the direction opposite to the gradient of the field strength:

$$
f=-\frac{\Delta \epsilon_{\perp}}{B} \nabla_{\|} B
$$


where $\epsilon_{\perp}=m_{e} v_{\perp}^{2} / 2$ is the gyration energy and $\Delta \epsilon_{\perp}$ denotes how much it changes in response to the heating. This extra mirror force acts to push the heated electrons in the direction of lower magnetic field strength, but if the heating is modulated on the time scale of the ion gyrofrequency, the ions do not have time to move. An electric field then arises to keep the plasma quasineutral by preventing the electrons from moving. The magnitude of this field can be estimated by balancing the electric force with the extra mirror force, resulting in

$$
E_{\|} \sim-\frac{\Delta \epsilon_{\perp} \nabla_{\|} B}{e B}
$$

where $-e$ denotes the electron charge. It should be specifically noted that collisional isotropisation of electron pressure would weaken the effect. The proposed mechanism works best in high temperature collisionless plasmas.

This single-particle estimate can be confirmed by a more careful analysis of the electron fluid equation of motion. Modulated ECRH causes the electron pressure tensor to be anisotropic,

$$
\mathbf{P}=p_{\|} \mathbf{b} \mathbf{b}+p_{\perp}(\mathbf{I}-\mathbf{b} \mathbf{b})
$$

and to vary with time. Here $\mathbf{b}=\mathbf{B} / B$ denotes the unit vector along the magnetic field, I the unit tensor, and the parallel and perpendicular pressures are given by

$$
\begin{aligned}
& p_{\|}=n T_{\|}=\mathrm{constant}, \\
& p_{\perp}=n T_{\perp}(\mathbf{r}, t),
\end{aligned}
$$

where we take the parallel temperature $T_{\|}$to remain constant on the short time scale of ECRH modulation and the perpendicular temperature $T_{\perp}(\mathbf{r}, t)$ to vary. Both perpendicular and parallel temperatures are kinetic. In the fluid equation for electron motion along the magnetic field,

$$
m_{e} n \mathbf{b} \cdot \frac{d \mathbf{V}}{d t}=-n e E_{\|}-\mathbf{b} \cdot(\nabla \cdot \mathrm{P}),
$$

the divergence of the pressure tensor is

$$
\nabla \cdot \mathbf{P}=\mathbf{b} \mathbf{b} \cdot \nabla\left(p_{\|}-p_{\perp}\right)+\left(p_{\|}-p_{\perp}\right)(\mathbf{b} \nabla \cdot \mathbf{b}+\mathbf{b} \cdot \nabla \mathbf{b})+\nabla p_{\perp},
$$

where $\nabla \cdot \mathbf{b}=-\nabla_{\|} \ln B$. The parallel force from the pressure gradient

$$
-\mathbf{b} \cdot(\nabla \cdot \mathrm{P})=-\nabla_{\|} p_{\|}-\left(p_{\perp}-p_{\|}\right) \nabla_{\|} \ln B
$$

thus becomes

$$
-\mathbf{b} \cdot(\nabla \cdot \mathrm{P})=-n\left(T_{\perp}-T_{\|}\right) \nabla_{\|} \ln B,
$$

reflecting the tendency of the mirror force to push charged particles in the direction of decreasing magnetic field strength. If the modulation is so rapid that the ions have no time to move, so that $\mathbf{V}=0$, an electric field arises to keep the plasma quasineutral and becomes equal to

$$
E_{\|}=-\frac{T_{\perp}-T_{\|}}{e} \nabla_{\|} \ln B
$$

The achievable voltage along the magnetic field is thus proportional to the electron pressure anisotropy caused by the ECRH modulation. This anisotropy will be limited by electron collisions and by transport along the magnetic field, which in a weakly collisional plasma occurs on the time scale $\omega_{t}^{-1} \sim L / v_{T e}$ on which an electron with 
thermal speed $v_{T e}$ moves the macroscopic distance $L$ characteristic of the modulated heating. The pressure anisotropy can thus be estimated to be

$$
p_{\perp}-p_{\|} \sim \frac{1}{\omega} \frac{d P}{d V}
$$

where $d P / d V$ denotes the absorbed ECRH power density and

$$
\omega=\max \left[\left|\omega_{1}-\omega_{2}\right|, \nu_{e}, \omega_{t}\right]
$$

the largest of the modulation frequency, collision frequency and the transit frequency. For typical conditions of W7-X plasmas in the high-mirror configuration and a modulation frequency of $30 \mathrm{MHz}$, the expected electric field ranges between $30 \mathrm{~V} / \mathrm{m}$ and $100 \mathrm{~V} / \mathrm{m}$ depending on the density. For a macroscopic distance of $L \sim 1 \mathrm{~m}$, this field would result in a potential difference of $30-100 \mathrm{~V}$.

\section{Wave excitation by electrostatic fluctuations}

The electrostatic oscillations with charge separation will of course generate an oscillating current along the magnetic field line, which in return will generate an oscillating poloidal magnetic field. When superimposed on the equilibrium magnetic field, this leads to an oscillating helical field, which will decay into two counterpropagating shear Alfvén waves along the magnetic field lines. (The compressional Alfvén wave, which is the other solution of the cold-plasma dispersion relation, cannot be excited since it requires a radial component of the oscillating magnetic field.) The shear Alfvén wave has a resonance at the ion cyclotron frequency. Its excitation is only possible if the frequency is below the ion cyclotron frequency. The wave can then propagate around the torus. If the frequency is above the ion cyclotron frequency, the wave is evanescent and only a local oscillation at the position of ECRH-power deposition occurs. The wave vector $\vec{k}=\vec{k}_{\perp}+k_{\|} \vec{b}$ is determined by the shear Alfvén wave dispersion relation:

$$
N_{L}^{2}=\frac{\omega_{p i}^{2}}{\left(\omega_{c i}-\omega\right) \omega_{c i}}
$$

where $N_{L}=c k_{\|} / \omega$ is the refraction index of the left-hand polarized shear Alfvén wave, $\omega_{p i}$ and $\omega_{c i}$ are the ion plasma and ion cyclotron angular frequencies, respectively. The excitation frequency can be adjusted to match the wave excitation with the k-spectrum of the current oscillation.

The scheme thus acts like an internal ICRH antenna inducing an alternating current inside the plasma. In contrast to conventional ICRH antennas, which use an oscillating current perpendicular to the magnetic field for compressional Alfven wave excitation [8], here the shear Alfvén wave is excited. This wave has left-hand-side polarization and therefore directly couples to the ion cyclotron motion. However, the energy which can be conveyed to ions is potentially limited by Manley-Rowe relations $[9,10]$ by approximately 1 per mille. Therefore, it is unlikely to use the scheme for ion-cyclotron plasma heating, but it can still be employed for generation of energetic minority ions.

\section{Possibility of detection}

There are a number of diagnostics which are able to detect waves in the ion cyclotron range of frequencies. Firstly, there are the ion cyclotron emission (ICE) probes. The 
dot-B probes which are typically used for the detection of ICE are not able to detect electrostatic fluctuations, however, but shielding currents will perturb the equilibrium magnetic field and might thus be visible by the probe.

The collective Thomson scattering (CTS) diagnostic is capable of detecting the product of three-wave mixing of the probing ECRH beam and the generated wave in the ion cyclotron frequency range. Moreover, if ion Bernstein waves are excited, they could be used for diagnosing the ion temperature and plasma composition by CTS with higher signal-to-noise ratios than are currently achievable [11].

\section{Conclusions and outlook}

A novel method for generating electrostatic fluctuations in magnetically confined plasmas with ECRH is proposed. The essence of the method is to employ two overlapping microwave beams of slightly different frequencies to generate a beat wave of much lower frequency. The resulting ECRH modulation creates an oscillatory longitudinal motion along the field since the magnetic field strength in general varies along the field lines. The heating modulation frequency, being equal to the frequency difference of the two gyrotrons, is easily controlled and can be set to match ion cyclotron frequency.

We also suggest a possible conversion mechanism of the electrostatic oscillations into shear Alfvén waves. The resulting left-hand polarized wave can couple its power directly to the cyclotron motion of ions.

This method may also have large implications for the diagnostic development, particularly CTS. If ion Bernstein waves are generated, this may be used for ion temperature and plasma composition measurements by CTS with high signal-to-noise ratio.

\section{Acknowledgments}

The authors would like to thank Prof. M. Thumm and Dr. G. Müller for inspiration. The authors are very grateful to Dr. R. Ochoukov for fruitful discussions.

\section{References}

[1] D. Moseev, H.P. Laqua, S. Marsen, N. Marushchenko, T. Stange, H. Braune, F. Gellert, M. Hirsch, U. Hoefel, J. Knauer, J.W. Oosterbeek, Y. Turkin, and The Wendelstein 7-X Team. Inference of the microwave absorption coefficient from stray radiation measurements in Wendelstein 7-X. Nuclear Fusion, 57(3):036013, mar 2017.

[2] A Fasoli, J.A Dobbing, C Gormezano, J Jacquinot, J.B Lister, S.E Sharapov, and A Sibley. Alfven eigenmode excitation by ICRH beat waves. Nuclear Fusion, 36(2):258-263, feb 1996.

[3] A. Fasoli, D. Borba, B. Breizman, C. Gormezano, R. F. Heeter, A. Juan, M. Mantsinen, S. Sharapov, and D. Testa. Fast particles-wave interaction in the alfvn frequency range on the joint european torus tokamak. Physics of Plasmas, 7(5):1816-1824, 2000.

[4] K. Sassenberg, M. Maraschek, P.J. Mc Carthy, W. Bobkov, M. Garca-Muoz, N. Hicks, V. Igochine, P. Lauber, S. Gnter, and ASDEX Upgrade Team. Icrh beatwave excited toroidicity induced alfvn eigenmodes in asdex upgrade. Nuclear Fusion, 50(5):052003, 2010.

[5] V.A. Flyagin, A.V. Gaponov, I. Petelin, and V.K. Yulpatov. The Gyrotron. IEEE Transactions on Microwave Theory and Techniques, 25(6):514-521, jun 1977.

[6] D Mueller, V Erckmann, M Thumm, R Wilhelm, D Dorst, and W Melkus. Control and modulation of gyrotron RF power for ECRH at $28 \mathrm{GHz}$ and $70 \mathrm{GHz}$. In 13th Symp. on Fusion Technology, page 811, Varese, 1984. 
[7] Hans Persson. Electric Field along a Magnetic Line of Force in a Low-Density Plasma. Physics of Fluids, 6(12):1756, dec 1963.

[8] T.H. Stix and W.R. Palladino. No Title. Phys. Fluids, 1:713, 1958.

[9] J. Manley and H. Rowe. Some General Properties of Nonlinear Elements-Part I. General Energy Relations. Proceedings of the IRE, 44(7):904-913, jul 1956.

[10] H. Rowe. Some General Properties of Nonlinear Elements. II. Small Signal Theory. Proceedings of the IRE, 46(5):850-860, may 1958 .

[11] G. A. Wurden, M. Ono, and K. L. Wong. Ion-temperature measurement via laser scattering on ion Bernstein waves. Physical Review A, 26(4):2297-2300, oct 1982. 